\title{
Sobrecarga en el cuidador familiar de adultos mayores con enfermedad crónica y su relación con características sociodemográficas y de salud propias y de la persona a su cuidado
}

\author{
Julia Lema, Ivonne Bernui \\ Instituto de Ética en Salud, Facultad Medicina, UNMSM
}

Objetivos: Determinar la relación entre las características socio demográficas y de salud propias del cuidador y del adulto mayor dependiente con enfermedad crónica, a quien cuida en el hogar, con su nivel de sobrecarga.

Diseño: Estudio cuantitativo, descriptivo, correlacional.

Institución: Instituto de Ética en Salud, Facultad Medicina, UNMSM.

Participantes: Cuidadores de adultos mayores del Centro Integral de Atención al Adulto Mayor (CIAM) de la Municipalidad de Pachacamac.

Intervenciones: Se aplicó formulario estructurado para variables sociodemográficas y de salud, y cuestionario de Zarit. Principales medidas de resultados: Sobrecarga de cuidador.

Resultados: La mayoría de cuidadores fue mujeres con edad promedio 52 años $\pm 10,5$, hijas de los adultos mayores, casadas y con nivel superior técnico. En promedio, cuidaban hace $5,4 \pm 5,6$ años y 13 horas al día $\pm 6,4$. Los adultos mayores fueron, en su mayoría, mujeres con edad promedio de 82 años $\pm 8,2$ y viudas, con hipertensión, diabetes, enfermedad vascular, demencia, otros. La mayoría de cuidadores tenía nivel de sobrecarga intenso y fuerte presencia de insuficiente ingreso económico, con sentimientos de ser la única persona que puede cuidarlo y que no lo está haciendo bien; que se ha afectado su salud, su vida social; disminuido el tiempo para sí mismo; agobiado por compatibilizar el cuidado con sus otras responsabilidades.

Conclusiones: No se encontró relación entre la sobrecarga del cuidador con las variables sociodemográficas y de salud propias y las del adulto mayor a quien cuida.

Palabras clave: Cuidador, adulto mayor, cuidador familiar.

\section{Validación de guía electrónica interactiva de modelos de porciones de frutas y verduras en adolescentes}

Doris Delgado, Margot Quintana, Sissy Espinoza

Universidad Nacional Mayor de San Marcos

Objetivos: Validar una guía electrónica interactiva (GEI) de modelos de porciones de frutas y verduras (FV), en adolescentes.

Diseño: Estudio descriptivo de validación de prueba diagnóstica.

Institución: Universidad Nacional Mayor de San Marcos.

Participantes: Nutricionistas, adolescentes.

Intervenciones: Entrevista en 31 adolescentes varones y mujeres, de 10 a 12 años de edad, en dos Instituciones Educativas de Callao y San Juan de Lurigancho, respectivamente. La entrevista estructurada a expertos se realizó en dos etapas, primero para validar fotografías de porciones de FV y segundo para validar el contenido de la GEl; luego, se aplicó un cuestionario de validación, que calificaba según escala de Likert: Muy de Acuerdo (MA), De acuerdo (DA), Regular (R), En desacuerdo (ED), Muy en desacuerdo (MD).

Principales medidas de resultados: Validación de la GEI de modelos de porciones de frutas y verduras para adolescentes.

Resultados: Los expertos estuvieron MA en la pertinencia, contenido, coherencia, utilidad de los mensajes, mientras que los juegos consideran que debe mejorarse, en ambiente y lenguaje, que coincidió con el menor puntaje (alfa Cronbach = $0,48)$ que le dieron los adolescentes a este aspecto; mientras que a los otros -atracción, comprensión e involucramientole dieron una mejor calificación (alfa Cronbach >0,8).

Conclusiones: Se logró validar en tres fases la GEI de FV para adolescentes.

Palabras clave: Guía electrónica interactiva, frutas, verduras, adolescente. 